\title{
Smoothing data association for target trajectory estimation in cluttered environments
}

\author{
Sufyan Memon, Taek Lyul Song ${ }^{*}$ and Tae Han Kim
}

\begin{abstract}
For heavily cluttered environments with low target detection probabilities, tracking filters may fail to estimate the true number of targets and their trajectories. Smoothing may be needed to refine the estimates based on collected measurements. However, due to uncertainties in target motions, heavy clutter, and low target detection probabilities, the forward prediction and the backward prediction may not be properly matched in the smoothing algorithms, so that the smoothing algorithms may fail to detect the true target trajectories. In this paper, we propose a new smoothing algorithm to overcome such difficulties. This algorithm employs two independent integrated probabilistic data association (IPDA) tracking filters: one running forward in time (fIPDA) and the other running backward in time (bIPDA). The proposed algorithm utilizes bIPDA multi-tracks in each fIPDA path track for fusing through data association to obtain the smoothing innovation in a fixed-lag interval. The smoothing innovation is used to obtain the smoothing data association probabilities which update the target trajectory state and the probability of target existence. The fIPDA tracks are updated after smoothing using the smoothing data association probabilities, which makes the fIPDA path tracks robust for maneuvering target tracking in clutter. This significantly improves the target state estimation accuracy compared to the IPDA. The proposed algorithm is called fixed-lag smoothing data association based on IPDA (FLIPDA-S). A simulation study shows that the proposed algorithm improves false track discrimination performance for maneuvering target tracking in clutter.
\end{abstract}

Keywords: Smoothing data association, FLIPDA-S, False track discrimination, Target existence

\section{Introduction}

In target tracking, sensors detect targets as well as various unwanted objects in the surveillance area. Information about the targets' prior existence in the surveillance area is not known. The objects' sources are also unknown, with some possible sources being terrain reflections, thermal noise, and clouds. The unwanted objects are generally known as clutter. In a cluttered environment, the target measurements are present with a low probability of detection $P_{D}$ in each scan.

Tracks are initialized and updated using the measurements obtained in each scan. Track initialization in a cluttered environment results in both true tracks and

\footnotetext{
*Correspondence: tsong@hanyang.ac.kr

Department of Electronic System Engineering, Hanyang University, Ansan, Republic of Korea
}

false tracks. True tracks always follow the target measurements, whereas false tracks do not follow the target measurements. A technique called false track discrimination (FTD) is used to confirm the true tracks and terminate the false tracks. Almost all target-tracking algorithms employ track quality measures to achieve FTD. But the standard probabilistic data association (PDA) [1] does not provide a track quality measure for FTD. The multiple hypothesis tracker (MHT) [2] uses a sequential probability ratio as a track quality measure for FTD. In [3], the authors proposed a track-oriented MHT algorithm and applied a smoothing velocity vector for reduction of the false track establishment.

Integrated probabilistic data association (IPDA) $[4,5]$ introduces recursive formulae for data association and employs the probability of target existence as a track

\section{Springer}

(C) 2016 Memon et al. Open Access This article is distributed under the terms of the Creative Commons Attribution 4.0 International License (http://creativecommons.org/licenses/by/4.0/), which permits unrestricted use, distribution, and reproduction in any medium, provided you give appropriate credit to the original author(s) and the source, provide a link to the Creative Commons license, and indicate if changes were made. 
quality measure. The practical considerations for IPDA are discussed in [6]. IPDA is extended for maneuvering target tracking in [7] and [8]. The probability hypothesis density (PHD) algorithm $[9,10]$ estimates the number of targets and their trajectories without having the track-tomeasurement association. A multi-scan data associationbased integrated track splitting (ITS) algorithm [11, 12] updates the target state estimation in the current scan more accurately at the cost of increasing computational complexity. ITS also utilizes the probability of target existence as a track quality measure.

For target tracking in densely populated cluttered environments with a low probability of target detection, it becomes difficult to know the number of targets and their behavior in nature. In addition, the target trackers with autonomous track management function often fail to track the true targets in such environments. Smoothing may be needed to refine the tracking results. The smoothing algorithms use future scan information to improve the target state estimation accuracy in the current scan, at the cost of smoothing lag. The basic techniques of smoothing estimation are described in [13, 14], and [15]. A limited number of publications focus on smoothing target tracking. Augmented state fixed-lag smoothing based on the joint PDA (JPDA) algorithm is proposed in [16] for tracking multiple maneuvering targets in clutter. However, [16] does not provide a track quality measure for FTD. In [17], the authors applied IPDA to an augmented state system for fixed-lag smoothing and utilized the smoothing target existence probability as a track quality measure for FTD. In [18], the authors applied the fixed-interval Rauch-Tung-Striebel (RTS) $[14,15]$ smoothing formulae to the MHT algorithm to track a maneuvering target without a track quality measure for FTD. The fixed-interval smoothing IPDA (sIPDA) [19] uses the RTS smoothing formulae with the Fraser-Potter [20] approach to calculate the smoothing predictions and innovations.

In smoothing algorithms, the forward track prediction and the backward track prediction are based on measurements collected in a fixed-lag smoothing interval $[k, N]$. In the smoothing interval, the fusion of forward track prediction and backward track prediction is performed at scan $k$. Therefore, in an environment with densely populated clutter, low detection probabilities, and uncertain target motion, the forward predicted state and the backward predicted state may lie far away such that their contributions to establish the fused predicted state may be insignificant, which results in bad fusion performance. Due to this, fusion of forward prediction and backward prediction becomes a challenging issue. Thus, smoothing algorithm may fail to detect the true target trajectories. For such difficult environments, we propose a new smoothing algorithm called fixed-lag smoothing data association based on IPDA (FLIPDA-S). The proposed algorithm considers a constant-velocity model to deal with the problems of tracking a maneuvering target in a cluttered environment. A constant-velocity model is a dynamic model of a tracking filter that describes the target motion using a 4-dimensional state vector composed of 2-dimensional position and 2-dimensional velocity. The proposed work uses an arbitrary size of the smoothing interval $[k, N]$, where the first scan of each smoothing interval faces fixed-lag smoothing, such that the fixed-lag of the proposed algorithm is $N-k$. Unlike sIPDA [19], the proposed FLIPDA-S algorithm does not use the RTS algorithm for smoothing.

The proposed FLIPDA-S algorithm employs two independent IPDA filters, where the first IPDA filter runs in the forward (fIPDA) direction and the other runs in the backward (bIPDA) direction. The FLIPDA-S algorithm utilizes bIPDA multi-track state predictions. The backward multi-track state predictions at each scan in the smoothing interval are obtained recursively by utilizing the sensor measurements starting from the end of the smoothing interval to the beginning of the smoothing interval. The fIPDA tracks are initialized and maintained by using the sensor measurements at each scan. At each scan in the fixed-lag interval, the forward track state prediction of FLIPDA-S is used to produce smoothing innovations associating with the multiple tracks generated from bIPDA. In this fusion, each track in the forward path treats the backward path tracks as the measurements for data association. The smoothing data association probabilities, smoothed target trajectory state, and smoothed target existence probability are obtained by applying the smoothing innovations to the sensor measurements received at scan $k$ in the fixed-lag smoothing interval $[k, N]$. FLIPDA-S uses smoothing data association probabilities to update and propagate the existing forward tracks to the next smoothing interval. In the next fixed-lag interval, the FLIPDA-S uses each updated forward track and new forward tracks (initialized by fIPDA) to fuse with the backward path multiple tracks to smooth the target trajectory state and the probability of target existence. This procedure continues in each subsequent interval, which significantly improves both the target trajectory state estimation accuracy and the FTD. The simulation study of Section 5 shows improved FTD performance of FLIPDA-S as compared with sIPDA and IPDA for maneuvering target tracking in a cluttered environment.

The rest of this paper is organized as follows: The target and measurement models are discussed in Section 2. The fixed-lag smoothing data association algorithm based on IPDA (FLIPDA-S) is proposed in Section 3. Practical considerations of FLIPDA-S are presented in Section 4, followed by descriptions and results of the simulation in Section 5. This paper is concluded in Section 6. 


\section{Model dynamics}

This section describes the target model and the sensor model. In this paper, we assume that the sensor has infinite resolution.

\subsection{Target model}

The target existence event in scan $k$ is a random event and is denoted by $\chi_{k}$. If the target trajectory state at scan $k$ is denoted by $x_{k}$, then the target trajectory propagates by

$$
\boldsymbol{x}_{k}=\boldsymbol{F} \boldsymbol{x}_{k-1}+\boldsymbol{v}_{k-1}
$$

where $\boldsymbol{F}$ is the forward state propagation matrix and $\boldsymbol{v}_{k-1}$ is the zero-mean white Gaussian plant noise sequence with known covariance matrix $\boldsymbol{Q}$. Equation (1) can be rewritten for the backward trajectory state $\boldsymbol{x}_{k}^{\tau}$ propagation as

$$
\boldsymbol{x}_{k}^{\tau}=\boldsymbol{F}_{b} \boldsymbol{x}_{k+1}+\boldsymbol{v}_{k+1}
$$

where $\boldsymbol{F}_{b}$ is the backward state propagation matrix and $\boldsymbol{v}_{k+1}$ is the zero-mean white Gaussian backward plant noise sequence with known covariance matrix $\boldsymbol{Q}_{b}$. These matrices are expressed below:

$$
\begin{aligned}
& \boldsymbol{F}=\left[\begin{array}{cc}
\boldsymbol{I}_{2} & T \boldsymbol{I}_{2} \\
\boldsymbol{O}_{2} & \boldsymbol{I}_{2}
\end{array}\right], \\
& \boldsymbol{Q}=q\left[\begin{array}{cc}
\frac{T^{4}}{4} \boldsymbol{I}_{2} & \frac{T^{3}}{2} \boldsymbol{I}_{2} \\
\frac{T^{3}}{2} \boldsymbol{I}_{2} & T^{2} \boldsymbol{I}_{2}
\end{array}\right],
\end{aligned}
$$$$
\boldsymbol{F}_{b}=\boldsymbol{F}^{-1}
$$

and

$$
\boldsymbol{Q}_{b}=\boldsymbol{F}^{-1} \boldsymbol{Q F}^{-T}
$$

where $\boldsymbol{I}_{2}$ is the $2 \times 2$ identity matrix, $\boldsymbol{O}_{2}$ is the $2 \times 2$ null matrix, the scalar quantity $q$ is the target acceleration uncertainty, and $T$ is the sampling time.

\subsection{Sensor model}

The target measurement at each scan $k$ is

$$
z_{k}=\boldsymbol{H} \boldsymbol{x}_{k}+\boldsymbol{w}_{k}
$$

where $\boldsymbol{H}$ is the measurement matrix, and $\boldsymbol{w}_{k}$ is the zeromean white Gaussian measurement noise sequence with known covariance matrix $\boldsymbol{R}$.

In addition to target measurements, the sensor also returns clutter measurements, which follow Poisson distributions [21]. The clutter measurement density $\rho_{k, i}$ is a function of the measurement specified in the surveillance coordinate system, and it is assumed to be known or estimated [21].

The measurement set does not have prior knowledge about their sources. $Z_{k}$ is the measurement set received at scan $k$, and $m_{k}$ is the number of measurements in $Z_{k}$ received at scan $k$. Let $Z^{k}=\left\{Z_{k}, Z^{k-1}\right\}$ be the cumulative set of measurements from the initial scan to the current scan $k$. The measurement set $Z^{k-1}$ gives the measurement information from the initial scan to scan $k-1$. bIPDA filter uses sensor measurements $\boldsymbol{Z}^{k+}$ (where superscript $k+$ indicates the scan index starting from scan $N$ to scan $k$ in the smoothing interval) for backward track estimation. Let $Z^{k+}=\left\{Z_{k}, Z^{k+1+}\right\}$ be the cumulative set of measurements from scan $k+1$ to the current scan $k$. If the $i$ th measurement of $\boldsymbol{Z}_{k}$ is $\boldsymbol{z}_{k, i}$, then the clutter measurement density at $z_{k, i}$ is $\rho_{k, i} \equiv \rho\left(\boldsymbol{z}_{k, i}\right)$.

\section{Fixed-lag smoothing data association based on the IPDA algorithm (FLIPDA-S)}

In this section, we propose the fixed-lag smoothing data association algorithm based on IPDA (FLIPDA-S) with an arbitrary size of the smoothing interval $[k, N]$. FLIPDA-S smoothens the target trajectory estimation and the target existence probability at each scan in the fixed-lag smoothing interval.

The forward IPDA [4] initializes the new multiple forward tracks based on measurements $Z^{k}$ received in the first scan $k$ of the smoothing interval. FLIPDA-S uses IPDA [4] in the backward direction. In the backward path, new multiple backward tracks are initialized and updated using measurements $Z^{k+}$ received at each scan in the smoothing interval, ranging from scan $N$ to scan $k$ such that $Z^{k+}=\left\{Z_{k}, Z_{k+1}, \ldots, Z_{N}\right\}$. At each scan, FLIPDA-S fuses each forward track state prediction $p\left\{\boldsymbol{x}_{k} \mid \chi_{k}, \boldsymbol{Z}^{k-1}\right\}$ with backward path multiple track state predictions $p\left\{x_{k}^{\tau} \mid \chi_{k}^{\tau}, Z^{k+1+}\right\}_{\tau}$ using the data association procedure to obtain information fusion state predictions needed to calculate the smoothing innovations. In the fusion, the backward path tracks assume the role of measurements of the forward path tracks for data association. The information fusion state prediction is updated through data association probabilities to obtain the smoothing innovation, which is used for calculating smoothing data association probabilities and the smoothed target trajectory state. FLIPDA-S uses the smoothing data association probabilities to update the existing forward tracks at the first scan $k$ of the smoothing interval. Then, FLIPDA-S uses the updated forward track state estimations to propagate the existing forward tracks to the next smoothing interval $[k+1, N+1]$. The smoothed probability of target existence is also calculated at each scan. At the end of each smoothing interval, the fixed-lag scan (first scan of each interval) is selected to calculate FLIPDA-S statistics (true track confirmation rates and estimation errors), and a new scan is appended for the next smoothing interval. The procedure continues for each subsequent smoothing interval. 


\subsection{Forward IPDA (fIPDA)}

This section describes the initialization of fIPDA [4] tracks using sensor measurement $Z_{k}$ received in the first scan of every smoothing interval. fIPDA waits bIPDA for an arbitrary lag time in each interval to initialize each new forward track based on sensor measurements $Z_{k}$ received in each fixed-lag interval. The procedure is as follows.

\subsubsection{Notation}

$\hat{\boldsymbol{x}}_{k-1 \mid k-1}$ and $\hat{\boldsymbol{P}}_{k-1 \mid k-1}$ are the mean and covariance, respectively, of the forward track state estimate for scan $k-1$ conditioned on $\boldsymbol{Z}^{k-1}$. $\overline{\boldsymbol{x}}_{k \mid k-1}$ and $\overline{\boldsymbol{P}}_{k \mid k-1}$ are the mean and covariance, respectively, of the forward track state prediction for scan $k$ conditioned on $Z^{k-1} \cdot \chi_{k}$ is the target existence event in the forward track, $P\left\{\chi_{k-1} \mid Z^{k-1}\right\}$ is the probability of target existence for scan $k-1$ conditioned on $Z^{k-1}$, and $P\left\{\chi_{k} \mid Z^{k-1}\right\}$ is the probability of target existence for scan $k$ conditioned on $Z^{k-1}$.

\subsubsection{Forward track initialization}

Measurements of the first scan of two consecutive smoothing intervals are used to initialize new forward tracks (two point differencing [6]) with an initial probability of target existence $P\left\{\chi_{k-1} \mid Z^{k-1}\right\}$. Each forward track carries a unique forward track identification number.

\subsection{3 fIPDA track propagation}

The fIPDA recursion at scan $k$ starts with the updated track probability density function (pdf) $p\left\{x_{k-1} \mid \chi_{k-1}, Z^{k-1}\right\}$ at scan $k-1$. The fIPDA target existence probability follows the Markov chain one model [5] and propagates by

$$
P\left\{\chi_{k} \mid Z^{k-1}\right\}=p_{1,1} P\left\{\chi_{k-1} \mid Z^{k-1}\right\}
$$

where $p_{1,1}$ is the fIPDA target existence transition probability [5]. Note that in [5], the Markov chain two model is also described but it is useful for occluded target tracking, which is not the subject of this paper.

Each forward track propagates by using a Kalman filter propagation:

$$
\left[\overline{\boldsymbol{x}}_{k \mid k-1}, \overline{\boldsymbol{P}}_{k \mid k-1}\right]=\mathbf{K F}_{\mathrm{p}}\left(\hat{\boldsymbol{x}}_{k-1 \mid k-1}, \hat{\boldsymbol{P}}_{k-1 \mid k-1}, \boldsymbol{F}, \boldsymbol{Q}\right)
$$

where $\mathbf{K F}_{\mathrm{p}}$ is the Kalman filter propagation, and the forward track state prediction and its covariance matrix are given by

$$
\begin{aligned}
\overline{\boldsymbol{x}}_{k \mid k-1} & =\boldsymbol{F} \hat{\boldsymbol{x}}_{k-1 \mid k-1} \\
\overline{\boldsymbol{P}}_{k \mid k-1} & =\boldsymbol{F} \hat{\boldsymbol{P}}_{k-1 \mid k-1} \boldsymbol{F}^{T}+\boldsymbol{Q}
\end{aligned}
$$

\subsection{Backward IPDA (bIPDA)}

The proposed algorithm uses an IPDA [4] filter in the backward direction. The bIPDA procedure starts from the scan $N$ of the smoothing interval $[k, N]$, and the bIPDA tracks move backward until they reach scan $k$. The procedure is as follows.

\subsubsection{Notation}

Let $\tau \epsilon\left\{\tau_{1}, \tau_{2}, \ldots, \tau_{n}\right\}$ be the label of backward tracks. $\hat{\boldsymbol{x}}_{k+1 \mid k+1+}^{\tau}$ and $\hat{\boldsymbol{P}}_{k+1 \mid k+1+}^{\tau}$ are the mean and covariance of the backward track state estimate for scan $k+1$ conditioned on $\boldsymbol{Z}^{k+1+} . \overline{\boldsymbol{x}}_{k \mid k+1+}^{\tau}$ and $\overline{\boldsymbol{P}}_{k \mid k+1+}^{\tau}$ are the mean and covariance of backward track state prediction for scan $k$ conditioned on $Z^{k+1+}$. The backward track state estimate and its covariance for scan $k$ conditioned on $Z^{k+}$ are denoted by $\hat{\boldsymbol{x}}_{k \mid k+}^{\tau}$ and $\hat{\boldsymbol{P}}_{k \mid k+}^{\tau}$, respectively. $\chi_{k}^{\tau}$ is the target existence event in the backward path track, $P\left\{\chi_{k+1}^{\tau} \mid Z^{k+1+}\right\}$ is the backward probability of target existence for scan $k+1$ conditioned on $Z^{k+1+}$, and $P\left\{\chi_{k}^{\tau} \mid Z^{k+1+}\right\}$ is the backward probability of target existence for scan $k$ conditioned on $Z^{k+1+}$.

\subsubsection{Backward track initialization}

The backward tracks are initialized in each scan using sensor measurements. Each pair of measurements in consecutive scans may initialize a new backward track (two point differencing [6]) with an initial backward probability of target existence, $P\left\{\chi_{k+1}^{\tau} \mid Z^{k+1+}\right\}$. Therefore, there are no backward tracks in the last two scans of each smoothing interval. Each new backward track carries a unique backward track identification number.

\subsubsection{Backward track propagation}

The bIPDA recursion at scan $k$ starts with the updated track pdf, $p\left\{\boldsymbol{x}_{k+1}^{\tau} \mid \chi_{k+1}^{\tau}, Z^{k+1+}\right\}$ at scan $k+1$. The bIPDA probability of target existence follows the Markov chain one model [5] and propagates by

$$
P\left\{\chi_{k}^{\tau} \mid Z^{k+1+}\right\}=p_{1,1}^{b} P\left\{\chi_{k+1}^{\tau} \mid Z^{k+1+}\right\}
$$

where $p_{1,1}^{b}$ is the bIPDA target existence transition probability [5]. Each backward track propagates by the Kalman filter propagation:

$\left[\overline{\boldsymbol{x}}_{k \mid k+1+}^{\tau}, \overline{\boldsymbol{P}}_{k \mid k+1+}^{\tau}\right]=\mathbf{K F}_{\mathrm{P}}\left(\hat{\boldsymbol{x}}_{k+1 \mid k+1+}^{\tau}, \hat{\boldsymbol{P}}_{k+1 \mid k+1+}^{\tau}, \boldsymbol{F}_{b}, \boldsymbol{Q}_{b}\right)$

where $\mathbf{K F}_{\mathrm{p}}$ is the Kalman filter propagation, and the backward track state prediction and its covariance matrix are expressed by

$$
\begin{aligned}
& \overline{\boldsymbol{x}}_{k \mid k+1+}^{\tau}=\boldsymbol{F}_{b} \hat{\boldsymbol{x}}_{k+1 \mid k+1+}^{\tau} \\
& \overline{\boldsymbol{P}}_{k \mid k+1+}^{\tau}=\boldsymbol{F}_{b} \hat{\boldsymbol{P}}_{k+1 \mid k+1+}^{\tau} \boldsymbol{F}_{b}^{T}+\boldsymbol{Q}_{b}
\end{aligned}
$$




\subsection{4 bIPDA measurement selection}

At each scan $k$ in the smoothing interval, each backward track selects a subset of validated measurements $z_{k, i}^{b}$ from the measurement set $Z_{k}$ received at scan $k$. A measurement selection procedure [5] is used to select the validated measurements $\boldsymbol{z}_{k, i}^{b}$ for each backward track. The validation measurements selection criterion is given by

$$
\boldsymbol{\Delta}_{i}^{k+1+} \boldsymbol{S}_{k+1+}^{-1} \boldsymbol{\Delta}_{i}^{k+1+} \leq g
$$

where $\boldsymbol{\Delta}_{i}^{k+1+}$ and $\boldsymbol{S}_{k+1+}$ denote the measurement $\boldsymbol{z}_{k, i}$ innovation and its covariance with respect to each backward track:

$$
\begin{gathered}
\boldsymbol{\Delta}_{i}^{k+1+}=\boldsymbol{z}_{k, i}-\boldsymbol{H} \overline{\boldsymbol{x}}_{k \mid k+1+}^{\tau} \\
\boldsymbol{S}_{k+1+}=\boldsymbol{H} \overline{\boldsymbol{P}}_{k \mid k+1+}^{\tau} \boldsymbol{H}^{T}+\boldsymbol{R}
\end{gathered}
$$

and $g$ is the validation selection threshold, which depends on the gating probability $P_{G}[6,22]$. In the 2-dimensional surveillance situation, $g$ is selected as 9.21, which corresponds on the gating probability $P_{G}=0.99$. The validated measurement $z_{k, i}^{b}$ selected by each backward track is defined by

$$
\boldsymbol{z}_{k}^{b}=\left\{\boldsymbol{z}_{k} ; \boldsymbol{\Delta}_{i}^{k+1+}{ }^{T} \boldsymbol{S}_{k+1+}^{-1} \boldsymbol{\Delta}_{i}^{k+1+} \leq g\right\}
$$

\subsection{5 bIPDA measurement likelihood}

At each scan $k$, each backward track calculates the measurement likelihood of validated measurement $z_{k, i}^{b}$ by [4]

$$
\begin{aligned}
& p_{k, i}^{b}= p\left(\boldsymbol{z}_{k, i}^{b} \mid k+1+, \boldsymbol{Z}^{k+1+}\right)= \\
&\left\{\begin{array}{cr}
\frac{1}{P_{G}} \mathcal{N}\left(\boldsymbol{z}_{k, i}^{b} ; \boldsymbol{H} \overline{\boldsymbol{x}}_{k \mid k+1+}^{\tau}, \boldsymbol{H} \overline{\boldsymbol{P}}_{k \mid k+1+}^{\tau} \boldsymbol{H}^{T}+\boldsymbol{R}\right) ; & \boldsymbol{z}_{k, i}^{b} \in \boldsymbol{Z}_{k} \\
0 ; & \boldsymbol{z}_{k, i}^{b} \notin \boldsymbol{Z}_{k}
\end{array}\right.
\end{aligned}
$$

\subsection{6 bIPDA track update}

The backward measurement likelihood ratio is expressed by

$$
\Lambda_{k}^{b}=1-P_{D} P_{G}+P_{D} P_{G} \sum_{i=1}^{m_{k}} \frac{p_{k, i}^{b}}{\rho_{k, i}}
$$

The updated bIPDA probability of target existence $[4,6]$ at scan $k$ is given by

$$
P\left\{\chi_{k+}^{\tau} \mid Z^{k+}\right\}=\frac{\Lambda_{k}^{b} P\left\{\chi_{k}^{\tau} \mid Z^{k+1+}\right\}}{1-\left(1-\Lambda_{k}^{b}\right) P\left\{\chi_{k}^{\tau} \mid Z^{k+1+}\right\}}
$$

At each scan $k$, each backward track state is updated by using the validated measurements $z_{k, i}^{b}$ for $i \geq 0$ $(i=0$ corresponds to the non-existence event). The data association probabilities corresponding to feasible track-to-measurement association events become

$$
\beta_{k, i}^{b}=\frac{1}{\Lambda_{k}^{b}}\left\{\begin{array}{l}
1-P_{D} P_{G} ; i=0 \\
P_{D} P_{G} \frac{p_{k, i}^{b}}{\rho_{k, i}} ; i>0
\end{array}\right.
$$

Each backward track state estimate is updated by applying $z_{k, i}^{b}$ in the Kalman filter update equation.

$\left[\hat{\boldsymbol{x}}_{k \mid k+, i}^{\tau}, \hat{\boldsymbol{P}}_{k \mid k+, i}^{\tau}\right]=\left\{\begin{array}{cl}{\left[\overline{\boldsymbol{x}}_{k \mid k+1+1}^{\tau}, \overline{\boldsymbol{P}}_{k \mid k+1+}^{\tau}\right] ;} & i=0 \\ \mathbf{K F}_{\mathrm{U}}\left(\boldsymbol{z}_{k, i}^{b}, \boldsymbol{R}, \overline{\boldsymbol{x}}_{k \mid k+1+}^{\tau}, \overline{\boldsymbol{P}}_{k \mid k+1+}^{\tau}\right) ; i>0\end{array}\right.$

where $\mathbf{K F}_{\mathrm{U}}$ is the Kalman filter estimation update defined by

$$
\begin{aligned}
\boldsymbol{S}_{k} & =\boldsymbol{H} \overline{\boldsymbol{P}}_{k \mid k+1+}^{\tau} \boldsymbol{H}^{T}+\boldsymbol{R} \\
\boldsymbol{K}_{k} & =\overline{\boldsymbol{P}}_{k \mid k+1+}^{\tau} \boldsymbol{H}^{T} \boldsymbol{S}_{k}^{-1} \\
\hat{\boldsymbol{x}}_{k \mid k+}^{\tau} & =\overline{\boldsymbol{x}}_{k \mid k+1+}^{\tau}+\boldsymbol{K}_{k}\left(\boldsymbol{z}_{k, i}^{b}-\boldsymbol{H} \overline{\boldsymbol{x}}_{k \mid k+1+}^{\tau}\right) \\
\hat{\boldsymbol{P}}_{k \mid k+}^{\tau} & =\overline{\boldsymbol{P}}_{k \mid k+1+}^{\tau}-\boldsymbol{K}_{k} \boldsymbol{H} \overline{\boldsymbol{P}}_{k \mid k+1+}^{\tau}
\end{aligned}
$$

Using (21), the Gaussian mixture [19] of updated backward track state estimate (22) becomes

$$
\hat{\boldsymbol{x}}_{k \mid k+}^{\tau}=\sum_{i=1}^{m_{k}} \beta_{k, i}^{b} \hat{\boldsymbol{x}}_{k \mid k+, i}^{\tau}
$$

and

$\hat{\boldsymbol{P}}_{k \mid k+}^{\tau}=\sum_{i=1}^{m_{k}} \beta_{k, i}^{b}\left(\hat{\boldsymbol{P}}_{k \mid k+, i}^{\tau}+\hat{\boldsymbol{x}}_{k \mid k+, i}^{\tau}\left(\hat{\boldsymbol{x}}_{k \mid k+, i}^{\tau}\right)^{T}\right)-\hat{\boldsymbol{x}}_{k \mid k+}^{\tau}\left(\hat{\boldsymbol{x}}_{k \mid k+}^{\tau}\right)^{T}$

\subsection{Information fusion state prediction and innovation}

At the first scan of each fixed-lag smoothing interval, the information fusion state prediction and innovation are formed by fusing each forward track prediction with backward path multi-track predictions falling in their validation region, as illustrated in Fig. 1.

Figure 1 shows an example of the fusion procedure, where $\circ$ indicates forward track prediction and $\times$ indicates backward track predictions inside the validation gate created by the forward prediction. The fusion is based on the following assumptions:

- the bIPDA multi-track prediction $p\left\{x_{k}^{\tau} \mid \chi_{k}^{\tau}, Z^{k+1+}\right\}$ (where, $\tau$ is the label of a backward track) received at scan $k$, assumes the role of a measurement to be used by the forward tracks.

- All backward tracks are mutually exclusive measurements. 


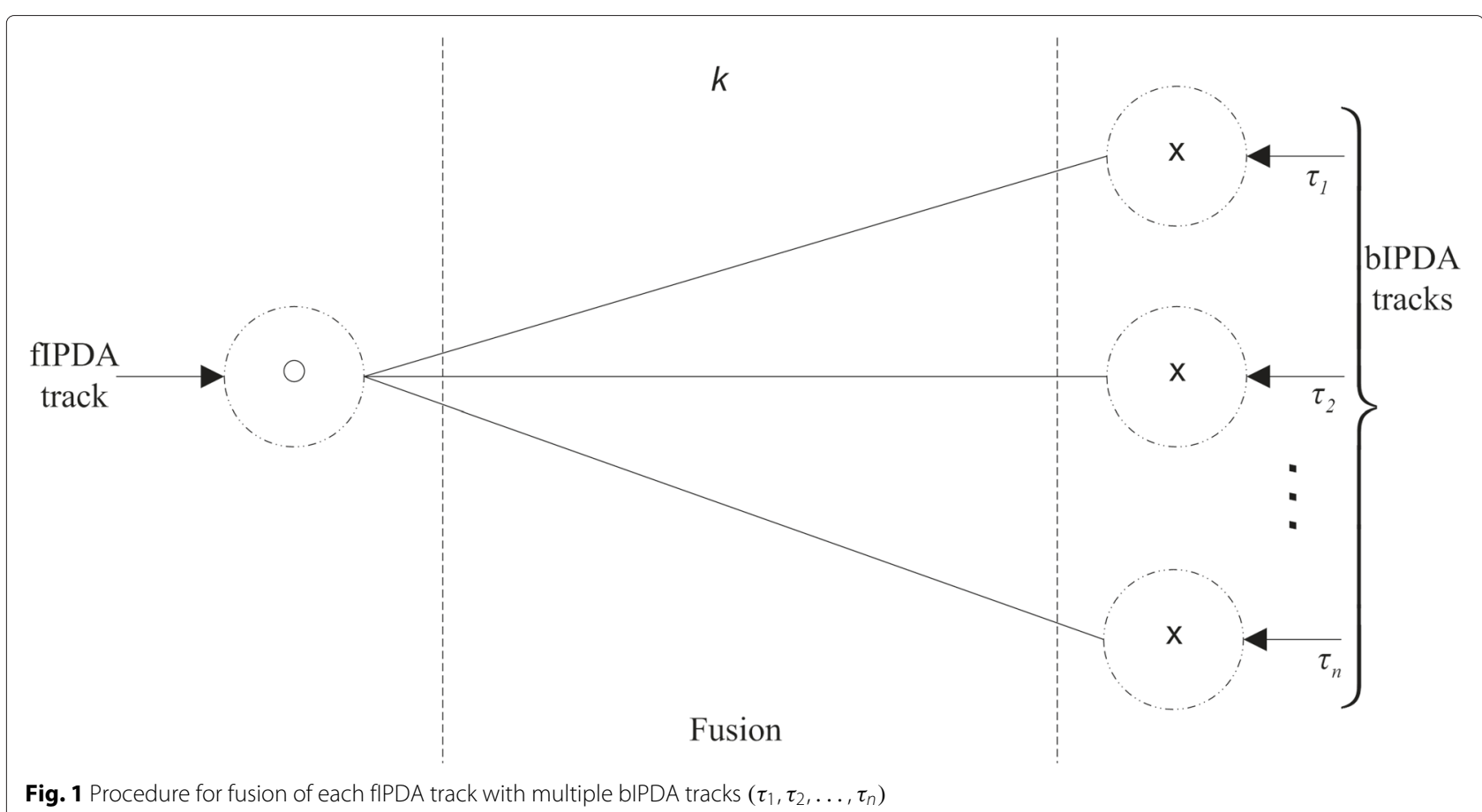

- $\left(1-P_{D}\right)^{N-k+1}$ is the non-detection probability (probability that a track does not exist in the interval $[k, N])$.

- $P_{D}^{*}=1-\left(1-P_{D}\right)^{N-k+1}$ is the probability that the track does exist in the interval $[k, N]$.

- The false track density of bIPDA satisfies

$$
\rho_{k, i}^{\tau} \approx \frac{n_{b}}{\mathbf{A}}
$$

where $\rho_{k, i}^{\tau}$ is the false track density of bIPDA, $n_{b}$ is the total number of backward false tracks inside the surveillance region, and $\mathbf{A}$ is the area of the surveillance region. The procedure is as follows.

\subsubsection{Information fusion measurement selection}

In the fusion, the bIPDA track predictions form a set of measurements at scan $k$. Each forward track prediction fuses with a subset of selected backward track predictions. A measurement selection procedure [5] is used to select the validated backward track predictions to ensure that the forward track prediction and the backward track prediction are properly matched. The measurement selection criterion is [5]

$$
\boldsymbol{\Delta}_{i}^{k^{T}} \boldsymbol{S}_{k}^{-1} \boldsymbol{\Delta}_{i}^{k} \leq g
$$

where $\boldsymbol{\Delta}_{i}^{k}$ and $\boldsymbol{S}_{k}$ represent the measurement innovation and its covariance with respect to each track $\tau$. Here,

$$
\boldsymbol{\Delta}_{i}^{k}=\boldsymbol{H} \overline{\boldsymbol{x}}_{k \mid k+1+}^{\tau}-\boldsymbol{H} \overline{\boldsymbol{x}}_{k \mid k-1}
$$

$$
\boldsymbol{S}_{k}=\boldsymbol{H}\left(\overline{\boldsymbol{P}}_{k \mid k+1+}^{\tau}+\overline{\boldsymbol{P}}_{k \mid k-1}\right) \boldsymbol{H}^{T}
$$

The backward tracks which satisfy (27) are said to be inside the validation gate and they form a set of validated measurements associated with the forward track. The information fusion state prediction is obtained by using the information fusion formulae $[13,23]$.

$\left[\bar{x}_{k}^{\eta}, \overline{\boldsymbol{P}}_{k}^{\eta}\right]=\left\{\begin{array}{cl}{\left[\overline{\boldsymbol{x}}_{k \mid k-1}, \overline{\boldsymbol{P}}_{k \mid k-1}\right] ;} & \text { if } \tau \text { is a false track } \\ \operatorname{IF}_{\mathrm{U}}\left(\overline{\boldsymbol{x}}_{k \mid k+1+}^{\tau}, \overline{\boldsymbol{x}}_{k \mid k-1}, \overline{\boldsymbol{P}}_{k \mid k+1+}^{\tau}, \overline{\boldsymbol{P}}_{k \mid k-1}, \boldsymbol{H}\right) ; & \text { if } \tau \text { is the true track }\end{array}\right.$

where $\mathbf{I F}_{\mathbf{U}}$ is the information fusion filter update, $\eta$ is the label of the fusion track, and

$$
\begin{aligned}
& \left(\overline{\boldsymbol{P}}_{k}^{\eta}\right)^{-1}=\left(\overline{\boldsymbol{P}}_{k \mid k-1}\right)^{-1}+\left(\overline{\boldsymbol{P}}_{k \mid k+1+}^{\tau}\right)^{-1} \\
& \overline{\boldsymbol{x}}_{k}^{\eta}=\overline{\boldsymbol{P}}_{k}^{\eta}\left[\left(\overline{\boldsymbol{P}}_{k \mid k-1}\right)^{-1} \overline{\boldsymbol{x}}_{k \mid k-1}+\left(\overline{\boldsymbol{P}}_{k \mid k+1+}^{\tau}\right)^{-1} \overline{\boldsymbol{x}}_{k \mid k+1+}^{\tau}\right] .
\end{aligned}
$$

\subsubsection{Measurement likelihood of selected measurement}

The measurement likelihood $p_{k}^{\eta}$ of the track predictions of fIPDA and bIPDA for the validated backward track $\tau$ becomes 
$p_{k}^{\eta}=\left\{\begin{array}{cc}\frac{1}{P_{G}} \mathcal{N}\left(\overline{\boldsymbol{x}}_{k \mid k+1+}^{\tau}, \overline{\boldsymbol{x}}_{k \mid k-1}, \overline{\boldsymbol{P}}_{k \mid k+1+}^{\tau}+\overline{\boldsymbol{P}}_{k \mid k-1}\right) ; & \text { if } \tau \text { is the true track } \\ 0 ; & \text { if } \tau \text { is a false track }\end{array}\right.$

\subsubsection{Information fusion filter update}

The measurement likelihood ratio $\Lambda_{k}^{*}$ of the validated measurements satisfies

$$
\Lambda_{k}^{*}=1-P_{D}^{*} P_{G}+P_{D}^{*} P_{G} \sum_{\eta=1}^{m_{k}^{\tau}} \frac{p_{k}^{\eta}}{\rho_{k}^{\tau}}
$$

where $m_{k}^{\tau}$ is the number of validated backward tracks.

Data association probabilities for the fusion tracks are calculated by

$$
\beta_{k}^{\eta}=\frac{1}{\Lambda_{k}^{*}}\left\{\begin{array}{l}
1-P_{D}^{*} P_{G} ; \text { if } \eta \text { is a false fusion track } \\
P_{D}^{*} P_{G} \frac{p_{k}^{\eta}}{\rho_{k}^{\tau}} ; \text { if } \eta \text { is the true fusion track }
\end{array}\right.
$$

The Gaussian mixture [19] of (30) can be obtained by using the data association probabilities of (35) as

$$
\overline{\boldsymbol{x}}_{k \mid N \backslash k}=\sum_{\eta=1}^{m_{k}^{\tau}} \beta_{k}^{\eta} \overline{\boldsymbol{x}}_{k}^{\eta}
$$

and

$$
\overline{\boldsymbol{P}}_{k \mid N \backslash k}=\sum_{\eta=1}^{m_{k}^{\tau}} \beta_{k}^{\eta}\left(\overline{\boldsymbol{P}}_{k}^{\eta}+\overline{\boldsymbol{x}}_{k}^{\eta}\left(\overline{\boldsymbol{x}}_{k}^{\eta}\right)^{T}\right)-\overline{\boldsymbol{x}}_{k \mid N \backslash k}\left(\overline{\boldsymbol{x}}_{k \mid N \backslash k}\right)^{T}
$$

The FLIPDA-S target existence probability for the fusion step at scan $k$ is obtained by combining the predicted forward existence probability and measurement likelihood ratio obtained from the validated backward tracks. Using (8) and (34), the target existence probability for the fusion step is found to be

$$
P\left\{\chi_{k} \mid Z^{N \backslash k}\right\}=\frac{\Lambda_{k}^{*} P\left\{\chi_{k} \mid Z^{k-1}\right\}}{1-\left(1-\Lambda_{k}^{*}\right) P\left\{\chi_{k} \mid Z^{k-1}\right\}}
$$

where superscript $N \backslash k$ implies the interval composed of $[0, k-1] \cup[k+1, N]$.

\subsection{Fixed-lag smoothing IPDA (FLIPDA-S)}

At the first scan of the fixed-lag smoothing interval $[k, N]$, the FLIPDA-S track update is performed with the measurements inside the validation gate created by the fusion track represented by (36) and (37).

\subsubsection{FLIPDA-S measurement selection}

The validated measurements $z_{k, i}^{s}$ (where, superscript $s$ denotes the smoothing step) are obtained by applying (36) and (37) to a validation selection criterion.

$$
\Delta_{i}^{k \mid N \backslash k^{T}} \boldsymbol{S}_{k \mid N \backslash k}^{-1} \boldsymbol{\Delta}_{i}^{k \mid N \backslash k} \leq g
$$

where $\boldsymbol{\Delta}_{i}^{k \mid N \backslash k}$ and $\boldsymbol{S}_{k \mid N \backslash k}$ represent the innovation of $\boldsymbol{z}_{k, i}$ and its covariance, respectively, such as

$$
\begin{gathered}
\boldsymbol{\Delta}_{i}^{k \mid N \backslash k}=\boldsymbol{z}_{k, i}-\boldsymbol{H} \overline{\boldsymbol{x}}_{k \mid N \backslash k} \\
\boldsymbol{S}_{k \mid N \backslash k}=\boldsymbol{H} \overline{\boldsymbol{P}}_{k \mid N \backslash k} \boldsymbol{H}^{T}+\boldsymbol{R}
\end{gathered}
$$

\subsubsection{FLIPDA-S Measurement likelihood}

The smoothing likelihood measurement $p_{k, i}^{s}$ of $z_{k, i}^{s}$ for each fusion track satisfies

$$
\begin{aligned}
p_{k, i}^{s}= & p\left(z_{k, i}^{s} \mid Z^{N \backslash k}\right)= \\
& \left\{\begin{array}{cc}
\frac{1}{P_{G}} \mathcal{N}\left(z_{k, i}^{s} ; \boldsymbol{H} \overline{\boldsymbol{x}}_{k \mid N \backslash k}, \boldsymbol{H} \overline{\boldsymbol{P}}_{k \mid N \backslash k} \boldsymbol{H}^{T}+\boldsymbol{R}\right) ; & \boldsymbol{z}_{k, i}^{s} \in \boldsymbol{Z}_{k} \\
0 ; & \boldsymbol{z}_{k, i}^{s} \notin \boldsymbol{Z}_{k}
\end{array}\right.
\end{aligned}
$$

\subsubsection{FLIPDA-S update}

The FLIPDA-S measurement likelihood ratio $\Lambda_{k}^{s}$ at scan $k$ is

$$
\Lambda_{k}^{s}=1-P_{D} P_{G}+P_{D} P_{G} \sum_{i=1}^{m_{k}^{s}} \frac{p_{k, i}^{s}}{\rho_{k, i}}
$$

where $m_{k}^{s}$ is the number of the smoothing validated measurements.

The smoothed target existence probability $[4,6]$ can be obtained by using (38)

$$
P\left\{\chi_{k} \mid Z^{N}\right\}=\frac{\Lambda_{k}^{s} P\left\{\chi_{k} \mid Z^{N \backslash k}\right\}}{1-\left(1-\Lambda_{k}^{s}\right) P\left\{\chi_{k} \mid Z^{N \backslash k}\right\}}
$$

At scan $k$ of the fixed-lag smoothing interval $[k, N]$, each fusion track is updated by using smoothing validation measurements $\boldsymbol{z}_{k, i}^{s}$, where each feasible measurement outcome $i \geq 0$ generates a smoothing data association probability defined by

$$
\beta_{k, i}^{s}=\frac{1}{\Lambda_{k}^{s}}\left\{\begin{array}{l}
1-P_{D} P_{G} ; i=0 \\
P_{D} P_{G} \frac{p_{k, i}^{s}}{\rho_{k, i}} ; i>0
\end{array}\right.
$$

The FLIPDA-S smoothing track state is updated by using the validation measurements $z_{k, i}^{s}$ in the Kalman filter estimation update: 


$$
\left[\hat{\boldsymbol{x}}_{k \mid N, i}, \hat{\boldsymbol{P}}_{k \mid N, i}\right]=\left\{\begin{array}{cl}
{\left[\overline{\boldsymbol{x}}_{k \mid N \backslash k}, \overline{\boldsymbol{P}}_{k \mid N \backslash k}\right] ;} & i=0 \\
\mathbf{K F}_{\mathrm{U}}\left(\boldsymbol{z}_{k, i}^{s}, \boldsymbol{R}, \overline{\boldsymbol{x}}_{k \mid N \backslash k}, \overline{\boldsymbol{P}}_{k \mid N \backslash k}\right) ; & i>0
\end{array}\right.
$$

and the FLIPDA-S track output is a Gaussian mixture [19] of (46) and can be obtained by using (45).

$$
\hat{\boldsymbol{x}}_{k, N}=\sum_{i=1}^{m_{k}^{s}} \beta_{k, i}^{s} \hat{\boldsymbol{x}}_{k \mid N, i}
$$

and

$$
\hat{\boldsymbol{P}}_{k, N}=\sum_{i=1}^{m_{k}^{s}} \beta_{k, i}^{s}\left(\hat{\boldsymbol{P}}_{k \mid N, i}+\hat{\boldsymbol{x}}_{k \mid N, i}\left(\hat{\boldsymbol{x}}_{k \mid N, i}\right)^{T}\right)-\hat{\boldsymbol{x}}_{k, N}\left(\hat{\boldsymbol{x}}_{k, N}\right)^{T}
$$

where the subscript $k, N$ indicates that the calculated smoothed value for scan $k$ is conditioned on $Z^{N}$.

\subsection{Forward IPDA update for the next interval}

In this section, we describe how the FLIPDA-S algorithm uses the smoothing data association probabilities obtained in (45) to update and propagate each fIPDA track for the next smoothing interval.

\subsection{1 fIPDA measurement likelihood}

Each forward track calculates the measurement likelihood $p_{k, i}$ based on the smoothing validation measurement $z_{k, i}^{s}$ selected by (39).

$p_{k, i}=\left\{\begin{array}{cc}\frac{1}{P_{G}} \mathcal{N}\left(\boldsymbol{z}_{k, i}^{s} ; \boldsymbol{H} \overline{\boldsymbol{x}}_{k \mid k-1}, \boldsymbol{H} \overline{\boldsymbol{P}}_{k \mid k-1} \boldsymbol{H}^{T}+\boldsymbol{R}\right) ; & \boldsymbol{z}_{k, i}^{s} \in \boldsymbol{Z}_{k} \\ 0 ; & \boldsymbol{z}_{k, i}^{s} \notin \boldsymbol{Z}_{k}\end{array}\right.$

\subsection{2 fIPDA track update}

The forward track measurement likelihood ratio $\Lambda_{k}$ is defined by

$$
\Lambda_{k}=1-P_{D} P_{G}+P_{D} P_{G} \sum_{i=1}^{m_{k}^{s}} \frac{p_{k, i}}{\rho_{k, i}}
$$

The updated fIPDA probability of target existence at scan $k$ is $[4,6]$

$$
P\left\{\chi_{k} \mid Z^{k}\right\}=\frac{\Lambda_{k} P\left\{\chi_{k} \mid Z^{k-1}\right\}}{1-\left(1-\Lambda_{k}\right) P\left\{\chi_{k} \mid Z^{k-1}\right\}}
$$

At scan $k$ of the fixed-lag smoothing interval $[k, N]$, each forward track state is updated by using selected smoothing validation measurements $z_{k, i}^{s}$ for $i \geqslant 0$ such as

$$
\left[\hat{\boldsymbol{x}}_{k \mid k, i}, \hat{\boldsymbol{P}}_{k \mid k, i}\right]=\left\{\begin{array}{cc}
{\left[\overline{\boldsymbol{x}}_{k \mid k-1}, \overline{\boldsymbol{P}}_{k \mid k-1}\right] ;} & i=0 \\
\mathbf{K F}_{\mathrm{U}}\left(\boldsymbol{z}_{k, i}^{s}, \boldsymbol{R}, \overline{\boldsymbol{x}}_{k \mid k-1}, \overline{\boldsymbol{P}}_{k \mid k-1}\right) ; & i>0
\end{array}\right.
$$

and the smoothing data association $\beta_{k, i}^{s}$ obtained in (45) is used to update each forward track state estimation (52) by

$$
\hat{\boldsymbol{x}}_{k \mid k}=\sum_{i=1}^{m_{k}^{s}} \beta_{k, i}^{s} \hat{\boldsymbol{x}}_{k \mid k, i}
$$

and

$$
\hat{\boldsymbol{P}}_{k \mid k}=\sum_{i=1}^{m_{k}^{s}} \beta_{k, i}^{s}\left(\hat{\boldsymbol{P}}_{k \mid k, i}+\hat{\boldsymbol{x}}_{k \mid k, i}\left(\hat{\boldsymbol{x}}_{k \mid k, i}\right)^{T}\right)-\hat{\boldsymbol{x}}_{k \mid k}\left(\hat{\boldsymbol{x}}_{k \mid k}\right)^{T}
$$

The updated forward track estimation (based on the smoothing data association probabilities) is propagated to the next smoothing interval $[k+1, N+1]$, where the oldest target trajectory state (the first scan of a smoothing interval) is discarded and a new scan is appended. FLIPDA-S uses each existing updated forward track along with some new forward tracks (initialized by fIPDA) to fuse with multiple backward tracks in the next interval. Thus, the procedure is recycled to smooth the target trajectory state and the probability of target existence. This significantly improves target state estimation accuracy and FTD for smoothing maneuvering target trajectories as shown in Section 5 .

\section{Practical considerations}

This section presents practical considerations for the proposed work and its application in the simulation of Section 5.

\subsection{Smoothing delay time limit}

The smoothing target tracking algorithm has some predefined limited smoothing delay. The amount of smoothing delay depends on the application of the algorithm. A higher smoothing delay means that more measurements are available, which significantly reduces the estimation error. In this paper, a fixed-lag sliding smoothing interval procedure is used to limit the smoothing delay. In this case, the fixed-lag smoothing corresponds to only the first scan of each smoothing interval and to all the scans in the last smoothing interval in the simulation.

The smoothing interval $[k, N]$ consists of $N-k+1$ scans. The first scan of each smoothing interval is discarded, and a new scan is appended for the next smoothing interval, except the last smoothing interval. The FLIPDA-S 
statistics (the estimation errors and the true track confirmation rates) are accumulated for only the first scan of each smoothing interval, as well as for all the scans in the last smoothing interval. Let the current interval includes scans $l=3, \ldots, 7$. After the fixed-lag smoothing, the new interval contains $l=4, \ldots, 8$, and its fIPDA recursion starts at $k=4$, by using the fIPDA predicted track state, $p\left\{\boldsymbol{x}_{4} \mid \chi_{4}, \boldsymbol{Z}^{3}\right\}$, and the forward tracks are propagated after fixed-lag smoothing to the next interval. The new smoothing interval does not contain the existing backward tracks at the beginning of the new interval, and bIPDA initializes and updates the new tracks based on the measurements $Z_{k}$ received from scan $N$ to scan $k$.

\subsection{Track management}

The FLIPDA-S employs a track management procedure. Without track management, multiple fIPDA tracks and/or bIPDA tracks might follow the same target. The track management merges close tracks (following the same target) [6] and eliminates those tracks having lower probability of target existence. This approach is well defined in the literature and is omitted here.

\subsection{False track discrimination (FTD)}

FLIPDA-S provides the probability of target existence as a track quality measure. In the proposed algorithm, we apply the following FTD procedure:

- fIPDA tracks and bIPDA tracks are initialized for each smoothing interval.

- During the fIPDA and bIPDA procedures, each track is terminated if its updated probability of target existence falls below a predetermined termination threshold. This step eliminates the majority of false tracks.

- The updated FLIPDA-S probability of target existence is used to confirm and terminate tracks. FLIPDA-S tracks are confirmed when the updated FLIPDA-S probability of target existence exceeds a predetermined confirmation threshold and terminated when it falls below a predetermined termination threshold. The maximum smoothing is achieved at the first scan of each fixed-lag smoothing interval, once a FLIPDA-S track is confirmed.

- Each confirmed FLIPDA-S track stays confirmed until track termination.

\subsection{FLIPDA-S track output}

All confirmed tracks are used for output. Each output track consists of the FLIPDA-S trajectory estimate $\hat{\boldsymbol{x}}_{k, N}$, defined by (47). FLIPDA-S statistics generate only the results of confirmed true tracks, which must satisfy the true track test condition [19]. In this experiment, the following true track test condition [19] is used:

$$
\boldsymbol{\Delta}_{k, N}{ }^{T} \boldsymbol{P}_{2 \mid 2}^{-1} \boldsymbol{\Delta}_{k, N}<20
$$

where $\boldsymbol{\Delta}_{k, N}=\hat{x}_{k, N}-\boldsymbol{x}_{k}$ is the difference between the FLIPDA-S track state smoother and the true target trajectory state at scan $k$, and $\boldsymbol{P}_{2 \mid 2}$ is the track initialization covariance matrix [13];

$$
\boldsymbol{P}_{2 \mid 2}=\left[\begin{array}{cc}
\boldsymbol{R} & \boldsymbol{R} / T \\
\boldsymbol{R} / T & 2 \boldsymbol{R} / T^{2}
\end{array}\right]
$$

In addition, each confirmed track may become a false track if it meets the following false track test condition [19],

$$
\boldsymbol{\Delta}_{k, N}{ }^{T} \boldsymbol{P}_{2 \mid 2}^{-1} \boldsymbol{\Delta}_{k, N}>40
$$

\section{Simulations}

In this paper, the performance of FLIPDA-S is compared with the performance of SIPDA [19] and IPDA [4]. A maneuvering target trajectory in a cluttered environment is considered in the 2-dimensional surveillance area. The surveillance area is $800 \mathrm{~m}$ long along the $x$-axis and $600 \mathrm{~m}$ wide along the $y$-axis. The target trajectory state vector $x_{k}$ defined in (1) consists of 2-dimensional position and 2dimensional velocity vectors, such that $\boldsymbol{x}_{k}=[x, y, \dot{x}, \dot{y}]^{T}$. A linear sensor returns the target position measurements with probability of detection $P_{D}=0.8$ and sensor noise covariance $\boldsymbol{R}=25 \boldsymbol{I}_{2} \mathrm{~m}^{2}$. The clutter measurement density is equal to $1 \times 10^{-4} \mathrm{~m}^{-2}$. It is anticipated that all smoothing algorithms show similar performance for an environment with zero clutter measurement density.

The simulation consists of 1000 runs where each run has 48 scans. The target initial position is $[50 \mathrm{~m}, 200 \mathrm{~m}]^{T}$. The assumed maximum target velocity for track initialization [13] is $25 \mathrm{~m} / \mathrm{s}$. In the surveillance region, the target moves with a uniform motion of $15 \mathrm{~m} / \mathrm{s}$ for the first $24 \mathrm{~s}$ of the running time, and then the target maneuvers with a constant angular velocity of $\omega=0.06 \mathrm{rad} / \mathrm{s}$, until the end of the running time. The maneuvering dynamics of the target corresponding to the motion under the assumption of coordinated turn is defined by the following matrix [6].

$$
\boldsymbol{F}_{T}=\left[\begin{array}{cccc}
1 & 0 & \sin \omega T / \omega & -1-\cos \omega T / \omega \\
0 & 1 & 1-\cos \omega T / \omega & \sin \omega T / \omega \\
0 & 0 & \cos \omega T & -\sin \omega T \\
0 & 0 & \sin \omega T & \cos \omega T
\end{array}\right],
$$

Figure 2 shows the surveillance area and motion of the maneuvering target trajectory in clutter accumulated for one simulation run.

The FLIPDA-S uses Markov chain one model [5] of target existence for track initiation and propagation with the fIPDA state transition probability of $p_{1,1}=0.98$ and the bIPDA state transition probability of $p_{1,1}^{b}=1$.

Roughly 1,344,000 (28 per scan) false tracks are initialized per experiment. The work is done on the platform 


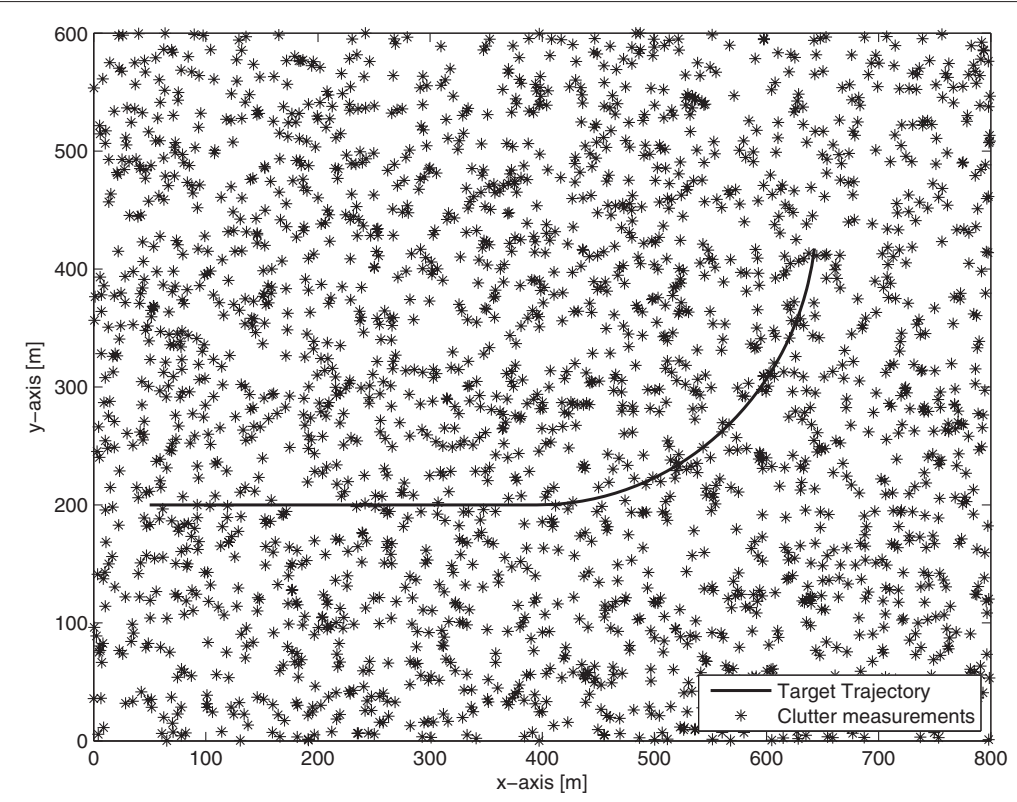

Fig. 2 Maneuvering target trajectory in clutter, inside the surveillance area

(Window 7, $2.8 \mathrm{GHz}$ Intel core 2 duo $\mathrm{CPU}$ and MATLAB R2008a), where IPDA, SIPDA, and FLIPDA-S have average execution time of $0.03 \mathrm{~s} / \mathrm{scan}, 0.08 \mathrm{sec} / \mathrm{scan}$, and $0.06 \mathrm{sec} / \mathrm{scan}$, respectively, for tracking a maneuvering target. The execution time for any track depends on many factors including the probability of detection, number of tracks, clutter measurement density, area of surveillance region, and track management procedure. In this environment, false tracks consume majority of computational resources. In addition, the computational complexity becomes twice if the number of targets and clutter measurements increase in the surveillance region. However, the proposed track management and FTD procedures terminate majority of false tracks and the efficiency of false track termination is reflected in the execution time.

Figures 3 and 4 show the performance (confirmed true track rates and smoothing position estimation errors) of FLIPDA-S with different smoothing lags. It is obvious that increasing the lag size improves smoothing performance as seen from reduction of the RMS estimation errors

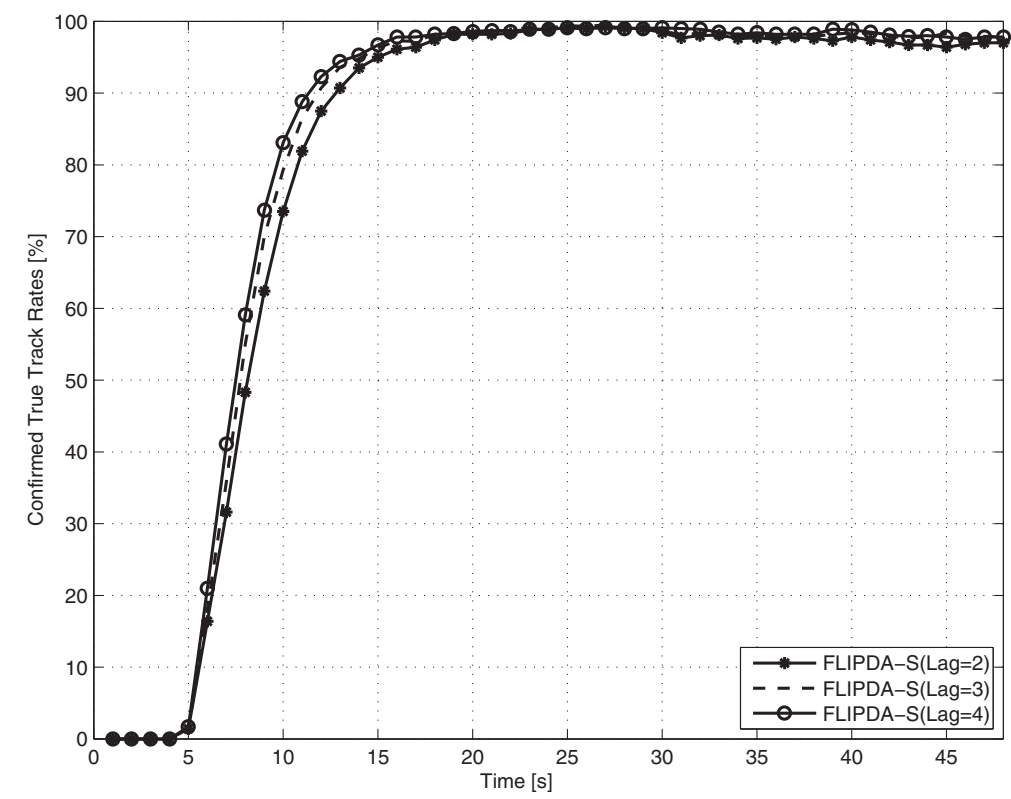

Fig. 3 Confirmed true track rates of FLIPDA-S (lag =4), FLIPDA-S $(\operatorname{lag}=3)$, and FLIPDA-S $(\operatorname{lag}=2)$ 


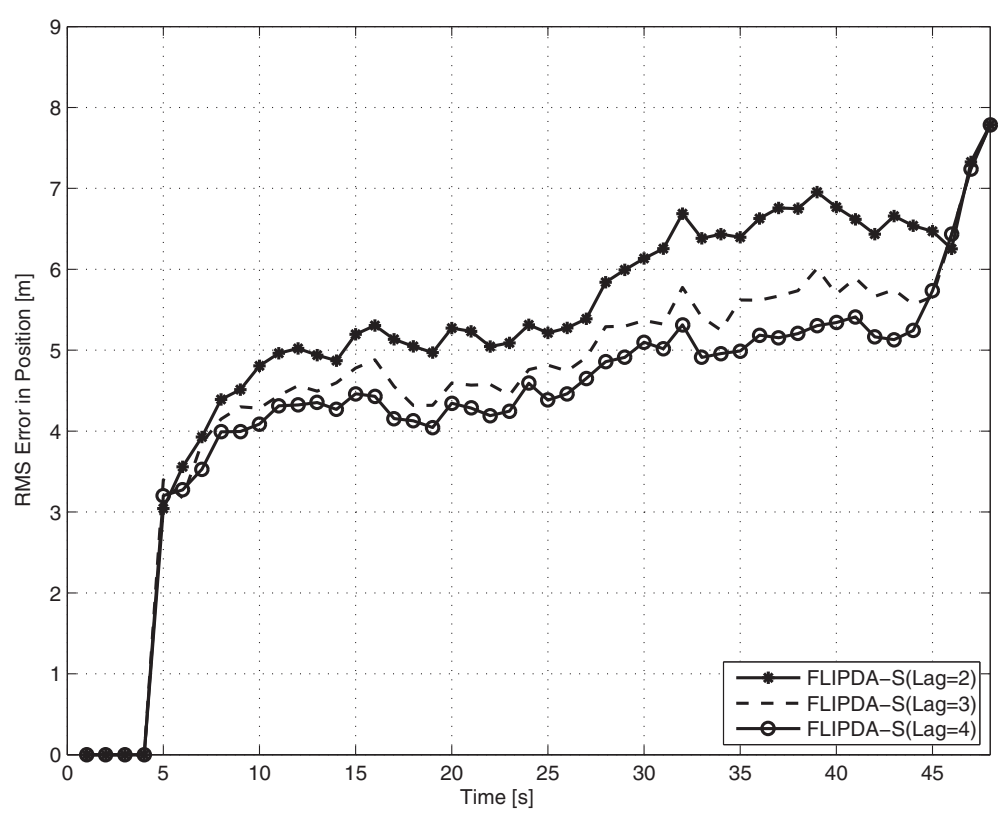

Fig. 4 Confirmed true track RMS position error of FLIPDA-S (lag =4), FLIPDA-S (lag =3), and FLIPDA-S (lag = 2)

and the true track management performance depicted in Figs. 3 and 4.

Both FLIPDA-S and sIPDA have the same smoothing interval, for a fair comparison. The initial probability of target existence for the algorithms IPDA, SIPDA, and FLIPDA-S are the same and the confirmation threshold for the algorithms are tuned to get the same number of confirmed false tracks $(\approx 68)$. Figure 5 shows confirmed false track rates of FLIPDA-S, sIPDA, and IPDA. In Fig. 5, the confirmed false track rates are evaluated as the sum of confirmed false tracks per scan received from entire simulation runs.

Figure 6 shows the true track confirmation rates of IPDA, sIPDA, and FLIPDA-S. Figure 6 provides a meaningful FTD comparison. When $k \leq N$, the FLIPDA-S and sIPDA state estimates are based on $Z^{N}$, and the IPDA state

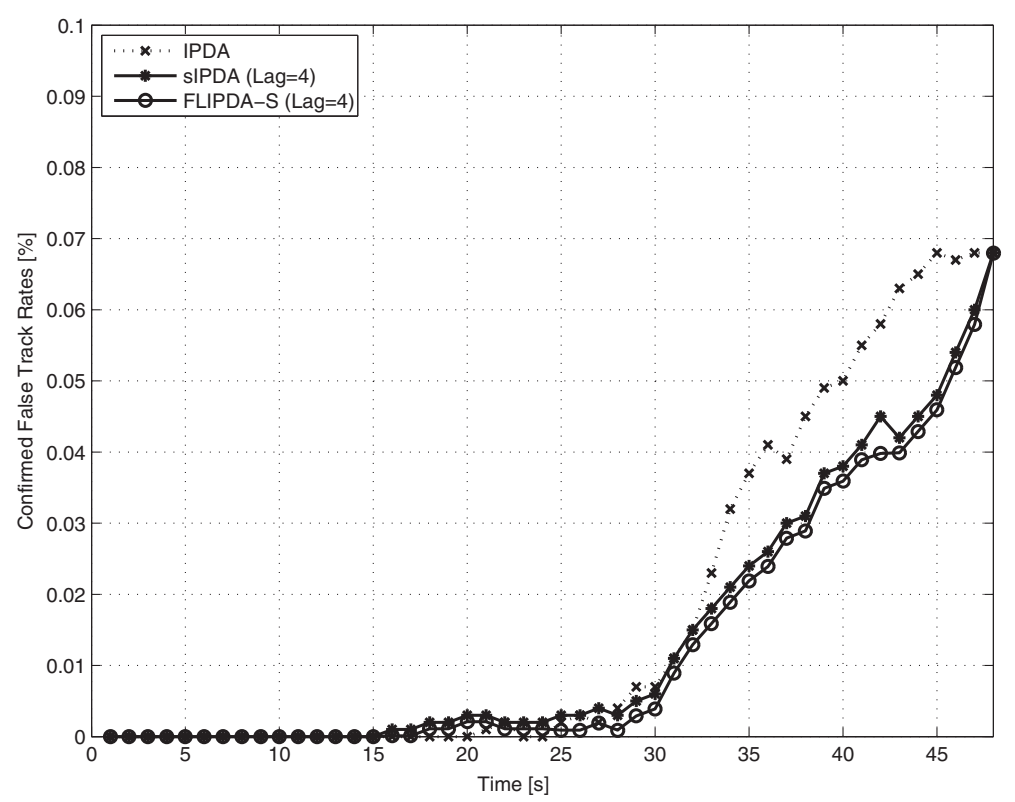

Fig. 5 Confirmed false track rates of FLIPDA-S $(\operatorname{lag}=4)$, SIPDA $(\operatorname{lag}=4)$, and IPDA 


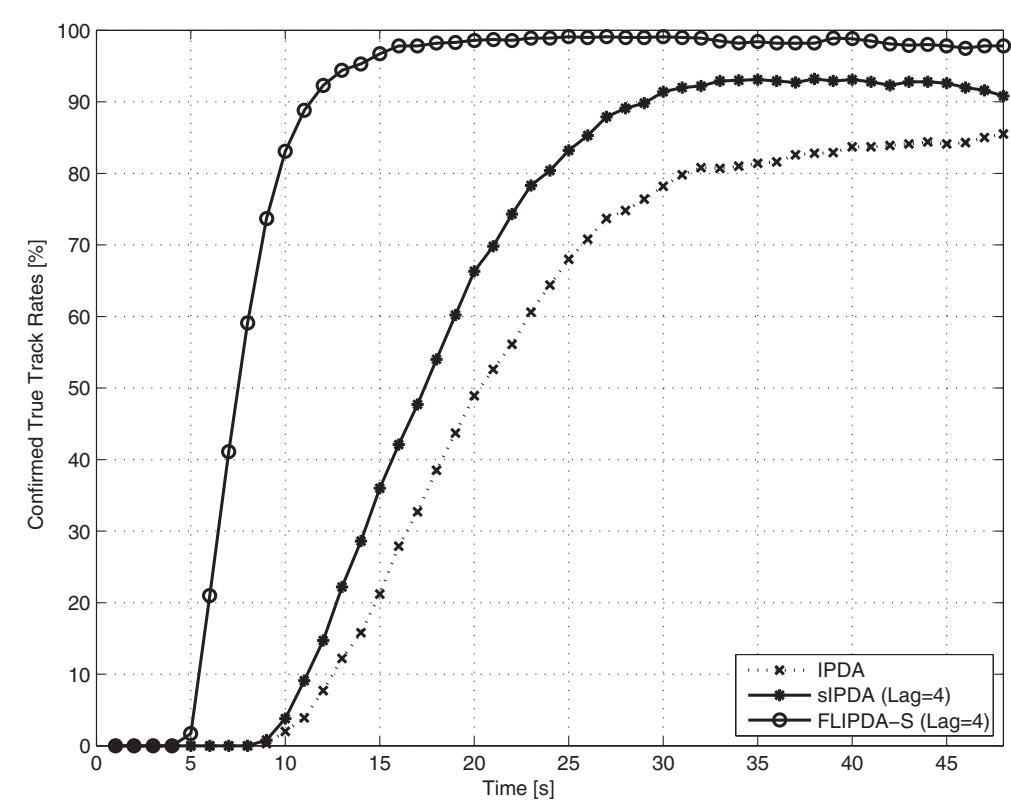

Fig. 6 Confirmed true track rates of FLIPDA-S (lag =4), sIPDA (lag =4), and IPDA

estimate is based on $Z^{k}$. Therefore, FLIPDA-S and sIPDA use more measurements than IPDA and have improved performance over IPDA. The smoothing data association used by FLIPDA-S provides fast buildup of the probability of target existence and has higher confirmation rate over sIPDA and IPDA, as shown in Fig. 6.

Figure 7 show the RMS error statistics (representing the position estimation errors of the trajectory state) of the confirmed true tracks of the algorithms in comparison. In the last scan of each simulation run, the FLIPDA-S, SIPDA, and IPDA state estimates are based on measurement $Z^{N}$ and the RMS errors of the confirmed true tracks of the algorithms converge to the same value. As mentioned in Section 3.2.2, the backward tracks do not exist in the last two scans of each smoothing interval. Note that the backward tracks of the proposed algorithm use less

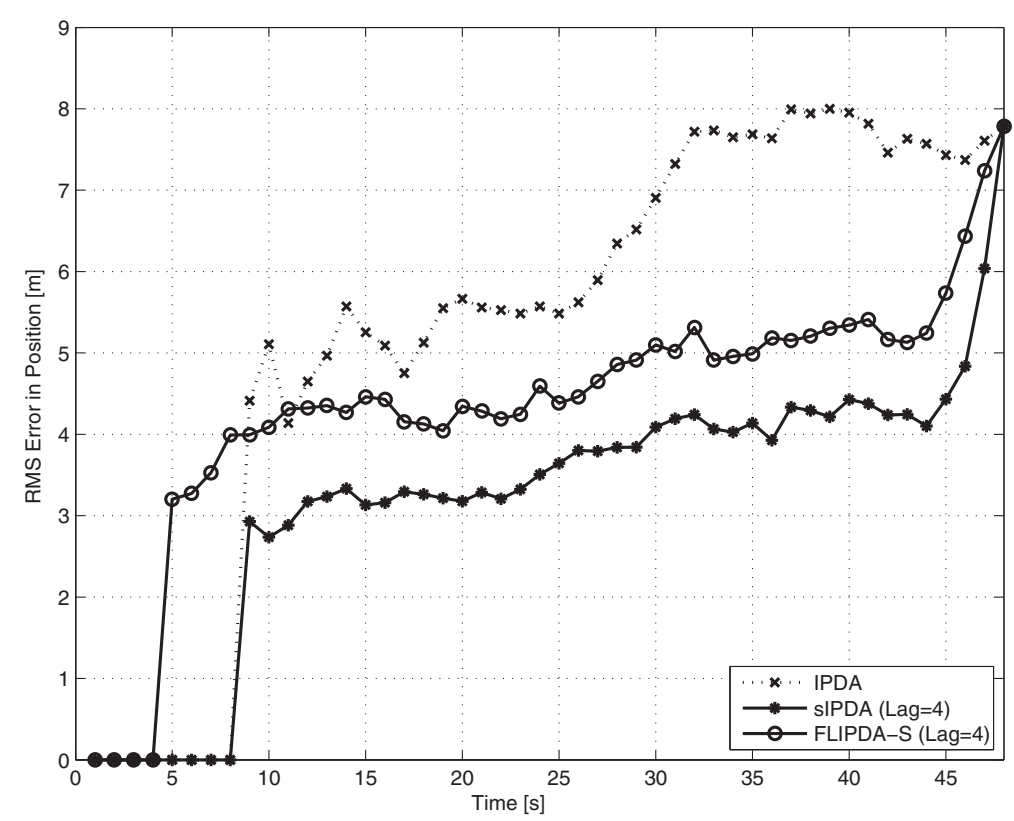

Fig. 7 Confirmed true track RMS position error of FLIPDA-S (lag $=4)$, sIPDA (lag $=4)$, and IPDA 
measurement information as compared to the measurements available for the backward tracks of sIPDA [19] in each smoothing interval. This results in the RMS error difference between FLIPDA-S and sIPDA as shown in Fig. 7. sIPDA, which is not a fixed-lag smoothing algorithm, utilizes the RTS equation for backward track generation, so that a backward track is generated for each forward track and it survives until the last scan of the smoothing interval. Therefore, the backward track is available from the last scan of the smoothing interval. Note also that as shown in Fig. 6, the number of confirmed true tracks of the proposed algorithm is much larger than that of SIPDA for RMS error statistics calculation in Fig. 7.

\section{Conclusions}

This paper presents a new smoothing algorithm called fixed-lag smoothing data association algorithm based on IPDA (FLIPDA-S) for tracking maneuvering targets in clutter using a constant-velocity model. At the first scan of each smoothing interval, the smoothing data association probabilities, the smoothing target trajectory estimation, and the smoothed target existence probability are obtained by using smoothing innovations. In the fusion, each fIPDA track prediction (including both the updated forward tracks based on smoothing data association probabilities and the new initialized forward tracks) fuses with the validated bIPDA track predictions through data association to obtain the information fusion state predictions, which is used for calculating the smoothing innovations. The simulation study shows a significant improvement in the target state estimation accuracy and FTD as compared to the IPDA. The FTD benefit of FLIPDA-S over SIPDA and IPDA is demonstrated by the effectiveness of applying smoothing data association algorithm to the tracking of maneuvering targets in clutter.

\section{Competing interests}

The authors declare that they have no competing interests.

\section{Acknowledgments}

This work was supported by the Agency for Defense Development, Korea (Grant UD130015DD).

Received: 7 September 2015 Accepted: 9 February 2016

Published online: 19 February 2016

\section{References}

1. Y Bar-Shalom, E Tse, Tracking in a cluttered environment with probabilistic data association. Automatica. 11, 451-460 (1975)

2. SS Blackman, Multiple hypothesis tracking for multiple target tracking. IEEE Trans. Aerospace Electronic Sys. 19(1), 5-18 (2004)

3. Y Obata, R Maekawa, M Ito, H Kamed, Y Kosuge, in ICCAS-SICE International Joint Conference. Tracking algorithm inheriting smoothing vector in splitting target tracking (IEEE, Fukuoka, 2009), pp. 3020-3025

4. D Mušicki, R Evans, S Stankovic, Integrated probabilistic data association. IEEE Trans. Autom. Control. 39(6), 1237-1241 (1994)

5. D Mušicki, Automatic tracking of maneuvering targets in clutter using IPDA PhD thesis. (University of Newcastle, Australia, 1994)

6. S Challa, R Evans, M Morelande, D Mušicki, Fundamentals of object tracking. (Cambridge University Press, New York, USA, 2011)
7. D Mušicki, S Suvorova, Tracking in clutter using IMM-IPDA-based algorithms. IEEE Trans. Aerospace Electronic Syst. 1(44), 111-126 (2008)

8. D Mušicki, S Challa, S Suvorova, in Asian Control Conference. Automatic track initiation of manoeuvring target in clutter (IEEE, Melbourne, Australia, 2004)

9. B-T Vo, B-N Vo, Bayesian filtering with random finite set observations. IEEE Trans. Signal Process. 56, 1313-1326 (2008)

10. TL Song, D Mušicki, Z Radosavljevic, Gaussian mixtures in multi-target tracking: a look at Gaussian mixture probability hypothesis density and integrated track splitting. IET Radar, Sonar Navigation. 6, 359-364 (2012)

11. D Mušicki, BFL Scala, RJ Evans, Integrated track splitting filter-efficient multi-scan single-target tracking in clutter. IEEE Trans. Aerosp. Electron. Syst. 43(4), 1409-1425 (2007)

12. D Mušicki, RJ Evans, Multiscan multitarget tracking in clutter with integrated track splitting filter. IEEE Trans. Aerosp. Electron. Syst. $\mathbf{4 5}$ 1432-1447 (2009)

13. B Anderson, J Moore, Optimal filtering. (Prentice-Hall Inc, Englewood Cliffs, New Jersey, 1979)

14. Y Bar-Shalom, XR Li, T Kirubarajan, Estimation with Applications to Tracking and Navigation: Theory Algorithms and Software. (Wiley-Interscience Publication, New York, USA, 2004)

15. TKailath, A Sayed, B Hasibi, Linear estimation. (Prentice-Hall Inc, Upper Saddle River, New Jersey, 2000)

16. C Bing, KT Jitendra, Tracking of multiple maneuvering targets in clutter using IMM/JPDA filtering and fixed-lag smoothing. Automatica. 37(2), 239-249 (2001)

17. U Khan, D Mušicki, TL Song, in Proceedings of 17th International Conference on Information Fusion. A fixed lag smoothing IPDA tracking in clutter (FUSION 2014, Salamanca, Spain, 2014)

18. W Koch, Fixed-interval retrodiction approach to Bayesian IMM-MHT for maneuvering multiple targets. IEEE Trans. Aerospace Electronic Sys. 36(1), 2-14 (2000)

19. TL Song, D Mušicki, Smoothing innovations and data association with IPDA. Automatica. 48(7), 1324-1329 (2012)

20. D Fraser, J Potter, The optimum linear smoother as a combination of two optimum linear filters. IEEE Trans. Autom. Control. 14(4), 387-390 (1969)

21. TL Song, D Mušicki, Adaptive clutter measurement density estimation for improved target tracking. IEEE Trans. Aerospace Electronic Sys. 2(47), 1457-1466 (2011)

22. Y Bar-Shalom, K Chang, H Blom, Multitarget Multisensor Tracking. (Artech House, Norwood, MA, 1990)

23. D Mušicki, TL Song, TH Kim, Smoothing multi scan target tracking in clutter. IEEE Trans. Signal Process. 61(19), 4740-4752 (2013)

\section{Submit your manuscript to a SpringerOpen ${ }^{\circ}$ journal and benefit from:}

- Convenient online submission

- Rigorous peer review

- Immediate publication on acceptance

- Open access: articles freely available online

- High visibility within the field

- Retaining the copyright to your article

Submit your next manuscript at springeropen.com 